\title{
Current status of therapy in nonalcoholic fatty liver disease
}

\author{
Scott McNear and Stephen A. Harrison
}

\begin{abstract}
The obesity epidemic has now spread worldwide. With increase in weight, there is an increase in dysregulated energy metabolism ultimately leading to dysfunction of multiple organ systems recognized as the metabolic syndrome. Nonalcoholic fatty liver disease (NAFLD) is the most common form of chronic liver disease worldwide, and is thought to be the hepatic manifestation of metabolic syndrome. It is a nondiscriminating disease affecting both children and adults and no socioeconomic class is spared. There is a well-defined increase in both liver-related and all-cause mortality. Current projections foresee a continued worsening in prevalence, especially with the increased rate of childhood obesity. Prevention would be the ultimate goal, but with continued trends in obesity, therapeutic options are needed to manage this chronic liver disease and prevent its complications of cirrhosis and even hepatocellular carcinoma. Therapies will need to be affordable, tolerable, and safe to be useful on such a large scale. This article will discuss some of the basic understanding of NAFLD, as well as review the currently tested therapies, some novel therapies, and potential future therapeutic options.
\end{abstract}

Keywords: nonalcoholic fatty liver disease, obesity, thiazolidinediones, metformin, HMG Co-A reductase inhibitors, ezetimibe, gemfibrozil, probucol, ursodeoxycholic acid

\section{Introduction}

The upward trend in obesity has become a significant global problem. The World Health Organization (WHO) has declared an epidemic that encompasses both industrialized and nonindustrialized nations. The fundamental cause of excess weight is a simple imbalance in calories consumed versus those expended. Major factors in this imbalance are a decrease in physical activity and the increased intake of energy-dense foods that are high in fats and sugars but low in essential vitamins and minerals. In 2005 the WHO projected 1.6 billion adults (age 15+) to be overweight $(\mathrm{BMI}>25)$ and 400 million to be obese $(\mathrm{BMI}>30)$. This problem is magnified with projections that by 2015 there will be 2.3 billion adults overweight with more than 700 million obese adults [WHO 2006]. Children are not immune to this problem, with upward trends in weight around the globe. Currently in the United States $25 \%$ of children are overweight and $11 \%$ are obese [Dehghan et al. 2005]. Sadly, it is estimated that $70 \%$ of obese adolescents will continue to be obese as adults [Parsons et al. 1999; Whitaker et al. 1997].
As the prevalence of obesity and metabolic syndrome (Table 1) has climbed on the global scale, so has the increase in nonalcoholic fatty liver disease (NAFLD). NAFLD is currently the most common form of chronic liver disease in the world with a prevalence estimated to be around $30 \%$ in the US [Browning et al. 2004]. The prevalence is significantly increased in diabetics (up to $63 \%$ ) and in the morbidly obese undergoing bariatric surgery (up to 93\%) [Gholam et al. 2007; Machado et al. 2006]. NAFLD is a nondiscriminate disease that may effect any age and demographic, with only a slight variation in risk. Within the spectrum of NAFLD, isolated fatty liver appears to be a rather benign disease with minimal risk of progression to advanced liver disease [Harrison et al. 2003a], but there has been an association with increased all-cause mortality, liver-related death and cardiovascular disease [Arslan et al. 2007; Targher et al. 2005]. Nonalcoholic steatohepatitis (NASH), on the other hand, is a more aggressive form of NAFLD with development of varying degrees of inflammation, fibrosis and possible progression to cirrhosis, end-stage liver disease and
Therapeutic Advances in Gastroenterology (2009) 2(1) 29-43 DOI: $10.1177 /$ 1756283X08100327

(C) The Author(s), 2009. Reprints and permissions: http://www.sagepub.co.uk/ journalsPermissions.nav

Correspondence to Stephen A. Harrison Chief of Hepatology, Brooke Army Medical Center, 3851 Roger Brooke Drive, Fort Sam Houston, TX 78234, USA stephen.harrisona amedd.army.mil Scott McNear Department of Medicine Brooke Army Medical Center, San Antonio, TX USA 
Table 1. Metabolic syndrome (Guidelines for diagnosis metabolic syndrome defined by World Health Organization in 1999).

Presence of diabetes mellitus, impaired glucose tolerance, impaired fasting glucose or insulin resistance, AND two of the following:

- Blood pressure: $\geq 140 / 90 \mathrm{mmHg}$

- Dyslipidaemia: triglycerides $\geq 1.695 \mathrm{mmol} / \mathrm{l}$ and high-density lipoprotein cholesterol $\leq 0.9 \mathrm{mmol} / \mathrm{l}$ (male), $\leq 1.0 \mathrm{mmol} / \mathrm{l}$ (female)

- Central obesity: waist:hip ratio $>0.9$ (male); $>0.85$ (female) and/or body mass index $>30 \mathrm{~kg} / \mathrm{m}^{2}$

- Microalbuminuria: urinary albumin excretion ratio $\geq 20 \mathrm{mg} / \mathrm{min}$ or albumin:creatinine ratio $\geq 30 \mathrm{mg} / \mathrm{g}$

hepatocellular carcinoma. The historical prevalence of NASH is $3-5 \%$ [Wanless and Lentz, 1990]; however, this is likely a current underestimation, as the prevalence of NASH has been shown to be $>35 \%$ in morbidly obese patients undergoing bariatric surgery [Machado et al. 2006].

\section{Diagnosis}

The evaluation and eventual diagnosis of NAFLD is commonly prompted by incidentally found abnormal serum aminotransferases or radiographic imaging suggesting fatty liver. Most patients do not have clinical symptoms that would prompt this specific evaluation. Diagnosis is based on the presence of steatosis without excessive ( $>20 \mathrm{~g} /$ day) alcohol consumption. Liver biopsy and imaging are currently the two modalities to aid in the diagnosis. Ultrasound, computed tomography or magnetic resonance studies can detect the presence of steatosis with a relatively high degree of accuracy [Joy et al. 2003]. All these modalities have their own strengths and weaknesses in regards to cost, availability, tolerability and potential adverse reaction. Ultrasound is less sensitive at detecting steatosis of less than 30\% [Mottin et al. 2004] and may ultimately require further imaging or liver biopsy to confirm diagnosis. Liver biopsy is currently the gold standard for diagnosis and staging of $\mathrm{NASH}$, as well as excluding other potential etiologies for liver disease. Liver biopsy delineates simple steatosis, or fat within the hepatocyte, from NASH that involves steatosis as well as hepatocyte inflammation, ballooning necrosis, and possibly perivenular/perisinusoidal fibrosis. These differences can have implications on the timing and therapies offered.

Interest has recently expanded in the development of noninvasive studies to delineate NASH with mild-to-moderate fibrosis from NASH with more severe fibrosis in an attempt to limit potentially unnecessary liver biopsies.
FibroTest-FibroSURE, a validated test for chronic hepatitis $\mathrm{C}$, uses six noninvasive markers to predict advanced fibrosis. This has been found to be useful as well in NAFLD with a specificity of $98 \%$ for advanced fibrosis [Ratziu et al. 2006]. Angulo et al. [2007] devised a NAFLD fibrosis score using six variables that included: age, BMI, hyperglycemia, platelet count, albumins and AST/ALT ratio, to identify NAFLD patients with and without advanced fibrosis with a positive predictive value of $82-90 \%$ and negative predictive value of $88-93 \%$ utilizing high and low cut-off scores. Wong et al. [2008] recently demonstrated similar results in the Chinese population with low prevalence of advanced fibrosis [Wong et al. 2008]. Further simplifying a clinical scoring system, the BARD score [Harrison et al. 2008], which is a four-point score system composed of BMI (one point), AST/ALT ratio of more than 0.8 (two points), and diabetes mellitus (one point) was created. A simply calculated score of 2 or greater is predictive of advanced fibrosis with an odds ratio of 17 and a negative predictive value of $96 \%$. Validation of these scoring systems and comparisons between them are needed however.

New imaging modalities such as transient elastography (Fibroscan; Echosens, Paris, France), magnetic resonance elastography [Manning and Afdhal, 2008] and contrast ultrasound (Lovovist; Shering, Berlin, German) [Iijima et al. 2007] have expanded our capability of detecting fibrosis. This technology has been shown to be very good at determining no/minimal fibrosis versus advanced disease, but is less sensitive for stages in between. Obesity is a current limitation for transient elastography due to interference by adipose tissue, but a newly designed probe may overcome this problem.

\section{Pathogenesis}

The pathological change of normal liver to that of steatosis is a complex process. Obesity, or more 
precisely increased visceral fat, has been shown to have a direct influence on glucose and lipid metabolism, with resultant liver steatosis and inflammation [van der Poorten et al. 2008]. Insulin resistance, leading to hyperinsulinemia, plays a significant, if not predominant role in the development of steatosis by increasing free fatty acid delivery to the liver, via increased intake and lipolysis, and increased de novo lipogenesis which contributing $60 \%$ and $26 \%$ of steatosis, respectively. Additionally, decreased free fatty acid oxidation and triacyglycerol export from the liver in the form of very-low-density lipoprotein make up the remaining causes [Donnelly et al. 2005]. More recent evidence has shown that macrophage infiltration in the visceral fat [Weisberg et al. 2003] contributes to insulin resistance and a cascade of events leading to upregulation of proinflammatory cytokines, which in turn decrease insulin signaling and adiponectin expression [Xu et al. 2003].

The development of liver inflammation and hepatic fibrosis found in some patients with NAFLD is less well understood. However, emerging data suggests that mitochondrial damage or dysfunction leads to an increase in reactive oxygen species which causes lipid peroxidation, further cytokine upregulation and the induction of Fas ligand [Das and Kar, 2005]. Lipid peroxidation causes a release of malondialdehyde and $\mu$-hydroxy-noneal and induces formation of specific cytokines such as tumor necrosis factor- $\alpha$, tissue growth factor- $\beta$ and interleukin-8. Fasligand expression can interact with Fas-ligand on other hepatocytes causing a cascade effect which can ultimately lead to apoptosis [Leevy, 1962]. Another important cause is the deficiency of the enzymes of perioxisomal $\beta$-oxidation leading to significant accumulation of dicarboxylic acids. Loss of these enzymes also leads to the sustained hyperactivation of perioxisome proliferation activated receptor- $\alpha$ regulated genes. Malondialdehyde and $\mu$-hydroxy-noneal may further lead to cell death, Mallory-Denk formation and promote collagen synthesis. It is also known now that the cytokines adiponectin and leptin play a significant role in steatosis and fibrosis [Huang et al. 2008; Weisberg et al. 2003; Haque and Sanyal, 2002]. As with most diseases, the understanding of the underlying biochemical dysfunction is vital to the prevention and treatment of that disease. Advances in our knowledge of the intricate balance between glucose and lipid metabolism, insulin and various other gut hormones, proinflammatory peptides, and their resultant effect on the liver have allowed for pathway specific medication to try and alter the course of this disease.

\section{Therapy}

Current therapies are targeted at the many different pathways that lead to the development of NAFLD. There is currently no US Food and Drug Administration-(FDA) approved medical therapy for the NAFLD. Therapeutic modalities (Figure 1; Table 2) are currently aimed at: (1) treatment of the metabolic conditions of diabetes and hyperlipidemia; (2) improving insulin sensitivity with medications, exercise and weight loss; (3) hepatoprotective medications to buffer oxidative stress, limit inflammatory cytokines and reduce fibrosis. Numerous studies have been conducted looking at all these options. Unfortunately most of these are limited with small enrollment, short duration of therapy, variable design, and lack of clear guidelines for treatment response. Larger, randomized, double-blind, placebo-controlled trials are needed to truly evaluate the efficacy and safety of these medications.

\section{Weight loss}

\section{Diet and exercise}

Weight loss achieved by reduced caloric intake and regular exercise has been shown to improve overall health and wellbeing. Various studies have proven that with moderate reduction in caloric intake, a significant improvement in serum aminotransferase levels $(p<0.05)$ as well as liver histology can be achieved [De Luis et al. 2008; Thomas et al. 2006; Huang et al. 2005]. Huang et al. demonstrated in a pilot study that intense nutritional counselling and a $1400 \mathrm{kcal} /$ day diet for 12 months could improve liver histopathology in $9 / 15$ patients. The average weight loss for improvement was $7 \%$ [Huang et al. 2005]. A similar result was found in a double-blind, placebo-controlled trial of 23 obese women comparing ursodeoxycholic acid and weight reduction with placebo and weight reduction. The study showed that weight loss alone was responsible for an improvement in biochemical $(p<0.001)$ and imaging score $(p<0.001)$ by ultrasound. An average of $2.5 \mathrm{~kg}, 7-8 \%$ body weight, was lost in both groups [Mendez-Sanchez et al. 2004]. Despite the improvements in these studies no significant change in fibrosis was seen in a 


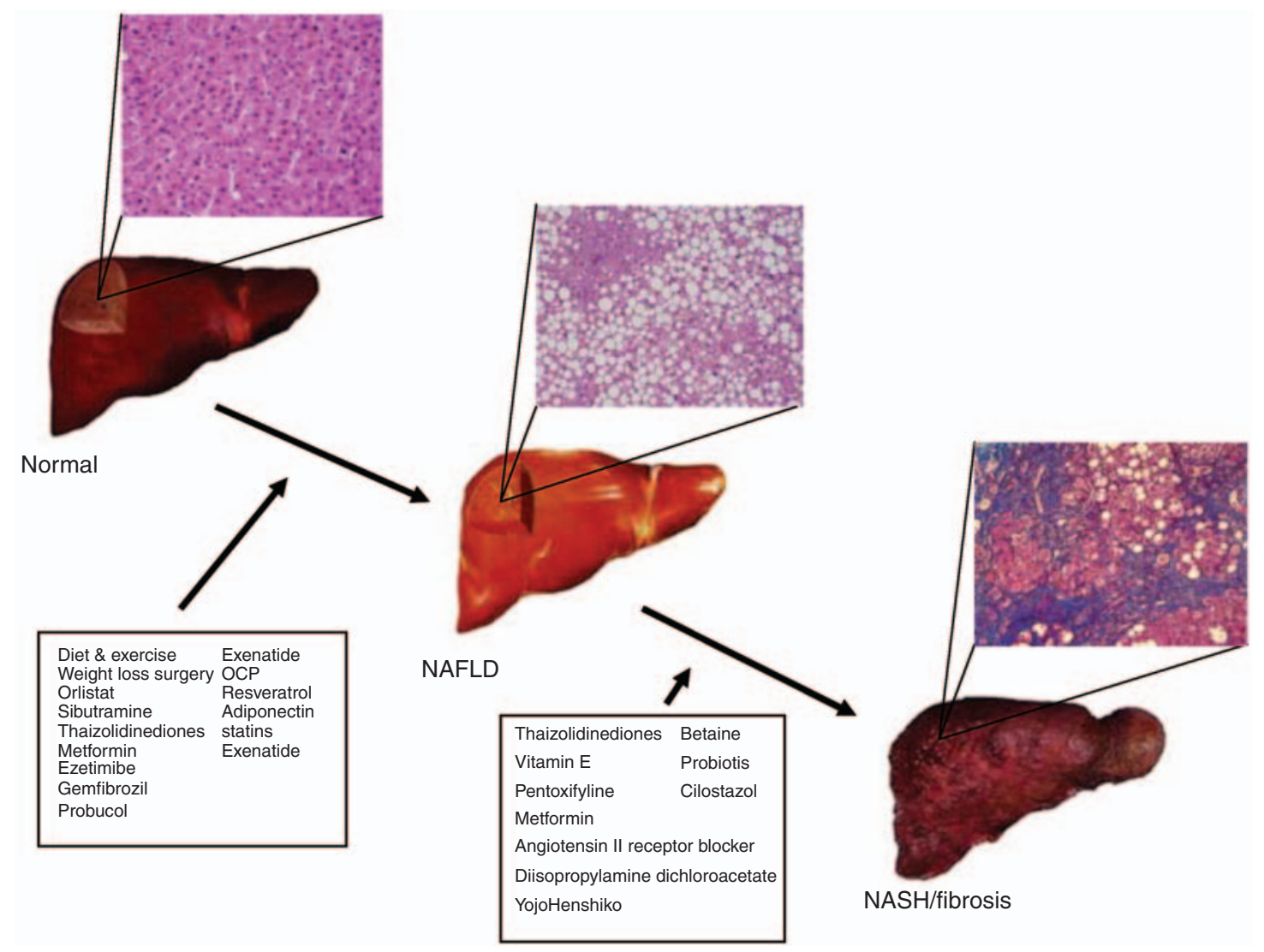

Figure 1. Therapeutic agents for nonalcoholic fatty liver disease.

meta-analysis of 15 studies for weight loss in the treatment of NAFLD [Wang and Koretz, 2003]. Extreme weight loss via starvation has been shown to worsen liver histology and fibrosis [Anderson et al. 1991].

The type of diet may have influence on treatment aside from caloric restriction. Zivkovic et al. [2007] examined the effect of popular weight loss diets for the treatment of NAFLD. The study showed benefit in diets that improved insulin sensitivity, promoted foods with low glycemic indices, and achieved sustained weight loss. This was demonstrated by Ryan et al. [2007] with a randomized, controlled trial comparing diets low in carbohydrate with higher carbohydrate/low fat diet. Despite equal weight loss in the two groups, the lower carbohydrate group had a more significant decline in alanine aminotransferase $(p<0.04)$. The overall decrease in insulin levels throughout the day appear to be a contributing factor to this benefit.

Sucrose and fructose have shown to have an effect on the development of NAFLD.
A population-based study showed that NAFLD patients consumed almost twice the amount of soft drinks containing fructose than the control counterparts [Ouyang et al. 2008; Zelber-Sagi et al. 2007]. High fructose consumption promotes increased de novo lipogenesis, hypertriglyceridemia, and hepatic insulin resistance [Le et al. 2007]. This concept has been further studied with dietary oligofructose, a nondigestible oligomer of $\beta$-D-fructose found in chicory root, artichoke, asparagus, and garlic. This has been shown to decrease serum and hepatic triglyceride production by decreasing expression of several lipogenic enzymes [Daubioul, 2000]. A small pilot study by Daubioul et al. [2004] of seven NASH patients showed a significant decrease in serum aminotransferases after 8 weeks of oligofructose therapy versus placebo $(p<0.05)$ [Daubioul et al. 2004]. Interestingly, a recent study by Bergheim et al. [2008] showed that administration of antibiotics could protect against fructose-induced hepatic injury in the murine model. The authors postulate that fructose dose not cause direct liver damage, but rather propagates intestinal translocation of 


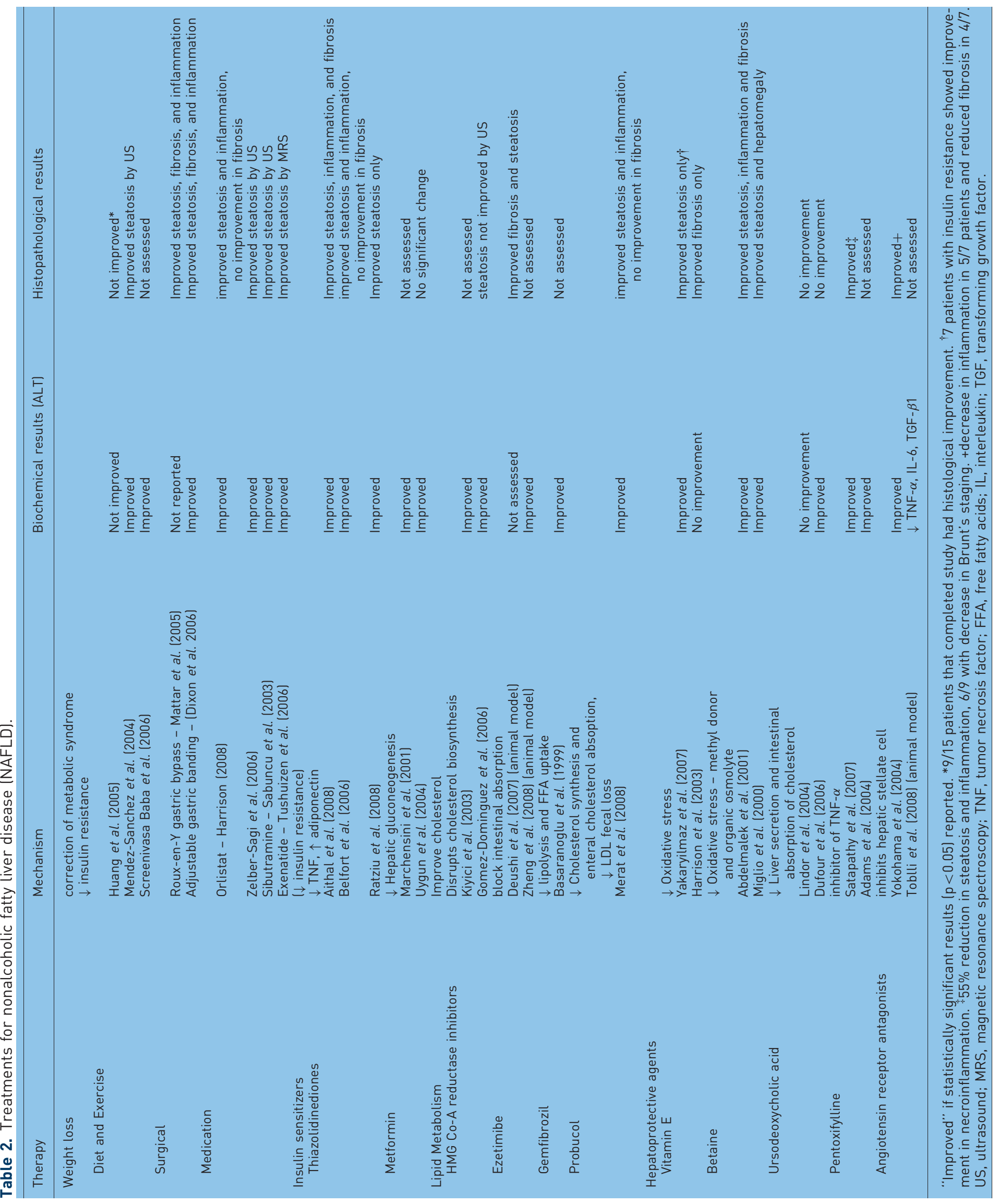


endotoxin with an increased inflammatory burden, resulting in fatty infiltration [Bergheim et al. 2008].

Exercise has also been shown to be a useful therapy for NAFLD. Information gathered to this point has been combination therapy with both exercise and dietary restriction. This combined approach has been shown to improve factors associated with metabolic syndrome as well as beneficial effects on NAFLD [Harrison et al. 2007; Screenivasa et al. 2006; Suzuki et al. 2005].

There is no clear consensus on the optimal diet or exercise regiment. It seems reasonable to aim for a decrease in 7-10\% body weight, possibly higher for the obese patient. A moderate exercise program three to four times a week should be encouraged to achieve a heart rate of $60-75 \%$ of the age-based maximum. Education should be provided on dietary changes with emphasis on low-glycemic-index foods and decreasing fructose consumption. These changes would best be initiated in a slow stepwise fashion to enhance patient compliance. Commercially available weight loss centers offer support and guidance and can be recommended. Further large studies may aid in optimal treatment strategies.

\section{Medications}

The most studied medication to promote weight loss is orlistat, a gastric and pancreatic lipase inhibitor. Two randomized controlled trials have shown a modest degree of weight loss as well as improvement in biochemical markers and hepatic steatosis and inflammation. One study showed a benefit of orlistat versus placebo despite equal amounts of weight loss [Zelber-Sagi et al. 2006]. A more recent study has shown that patients with $\geq 9 \%$ of body weight had significant improvement $(p<0.05)$ in aspartate aminotransferase, hepatic steatosis, inflammation and ballooning, as well as NAFLD Activity Score, but no significant change in fibrosis was seen [Harrison et al. 2007].

Sibutramine, an appetite suppressant, works on inhibiting central serotonin and norepinephrin reuptake. There has been only one study published looking at an effect on NASH. Thirteen patients achieved an average weight loss of $10.2 \%$ body weight, and improvement in liver enzymes (41-59\% reduction) and hepatic steatosis on ultrasound was found [Sabuncu et al. 2003].
However, this study was limited as there was no histopathologic follow up.

Glucagon-like protein 1 receptor agonists (incretin analogs), exenatide, have shown some benefit in the treatment of NAFLD. Initially designed for the treatment of diabetes, these agents promote insulin secretion, suppress inappropriate glucagon secretion, slow gastric emptying, and induce satiety. Modest weight loss can be seen due to these factors. A single case report showed improvement in serum alanine aminotransferase levels (46-20 IU/1) and hepatic steatosis by magnetic resonance spectroscopy, after 44 weeks of therapy [Tushuizen et al. 2006]. This medication targets many of the abnormalities associated with NAFLD, but it is currently a subcutaneous injection making it less appealing.

Another class of medication currently under investigation is rimonabant, a cannaboid-1 receptor inhibitor. This medication has been proven to reduce weight and waist circumference, and has had positive impact on triglyceride levels, insulin resistance, high-density lipoprotein levels, and adiponectin. There has also been improvement in biochemical levels and hepatic steatosis in an animal model [Gary-Bobo et al. 2007]. Two randomized, placebo-controlled trials are currently underway to evaluate for potential benefit in the NASH patients. Some safety concerns with depression have recently been addressed, and are currently limited to clinical research only in the US.

\section{Surgery}

Bariatric surgery has been shown to have the most significant effect on weight loss, correction of metabolic syndrome and treatment of NAFLD. Early surgeries with jejunoileal bypass resulted in significant weight loss, but also promoted significant worsening of liver disease [Marubbio et al. 1976]. More recent and newer surgeries such as Roux-en-Y gastric bypass and adjustable gastric banding have been shown to be better tolerated without resultant liver damage. A study of 70 obese patients underwent Roux-en-Y gastric bypass with a mean weight loss of $59 \%$ at 15 months and an $82 \%$ improvement in steatohepatitis and 39\% improvement in fibrosis [Mattar et al. 2005]. Adjustable gastric banding has had similar effects on steatosis, inflammation, and fibrosis $(p<0.001)$ [Dixon et al. 2006]. Long-term studies are needed to show stability of these findings however. In the meantime 
surgery, although more invasive, has become a very good therapeutic option for the treatment of NAFLD in morbidly obese patients without contraindications to surgery.

\section{Insulin sensitizers}

This group of medications is the most studied, due to the understanding that insulin resistance is the key component to the development of NAFLD. By improving glucose and insulin homeostasis, a critical step in the cascade of events resulting in the development of steatosis can be modified. However, a recent Cochrane review by Angelico et al. [2007] did not find any evidence to support or refute drugs aimed at influencing insulin resistance. There was noted improvement in serum aminotransferase and histology compared with placebo in various studies, but the studies pooled did not have enough power to give a definitive outcome.

\section{Thiazolidinediones}

This class of medication (pioglitazone and rosiglitazone) is an agonist of the peroxisome proliferator-activated receptor $\gamma$, which effects insulin resistance on skeletal muscle, adipose tissue and the liver by increasing adiponectin levels and fatty acid oxidation, as well as decreasing fatty acid synthesis. These medications have not been found to influence proinflammatory cytokines or leptin levels [Lutchman et al. 2006]. A randomized study by Sanyal et al. [2004] demonstrated a significant improvement in steatosis $(p<0.002)$, cytologic ballooning $(p<0.01)$, Mallroy's hyaline $(p<0.04)$, and pericellular fibrosis $(p<0.03)$ in 10 patients taking pioglitazone (30 mg/day) with vitamin E (400 IU/day) versus vitamin $\mathrm{E}$ alone [Sanyal et al. 2004]. A placebo-controlled trial of a hypocaloric diet with either pioglitazone ( $45 \mathrm{mg} /$ day) versus placebo showed similar results with improvement biochemical marker $(p=0.04$ to $<0.001)$ and liver histology with regard to steatosis $(p=0.003)$, ballooning necrosis $(p=0.02)$, and inflammation $(p=0.008)$. No significant change in fibrosis was seen [Belfort et al. 2006]. Aithal et al. [2008] further explored the benefit in 74 nondiabetic patients randomized to pioglitazone ( $30 \mathrm{mg} /$ day) or placebo for 12 months. This study showed, despite a slight weight gain, a significant decrease in alanine aminotransferase $(p=0.009)$ as well as improvement in liver histology with reduction in hepatocellular injury $(p=0.005)$, Mallory Denk bodies $(p=0.004)$, and fibrosis $(p=0.05)$. Recently released results of the 'FLIRT' trial showed improvement in biochemical markers $(p<0.005)$ and steatosis $(p<0.014)$, but no other significant improvement in liver histology [Ratziu et al. 2008]. These results may be explained by a study population of minimal histological damage at baseline, although the patients with more disease did have a significant improvement in histology. Based on the available data, these agents are likely have beneficial effect, but findings between the two studied thiazolidinediones have not been consistent possibly due to differences in study design and disease severity at baseline.

\section{Metformin}

Metformin, a biguanide, has been used for several decades for the treatment of diabetes by decreasing hepatic gluconeogenesis, limiting triacylglycerol production, and enhancing insulin sensitivity. It is a rather inexpensive medication, is well tolerated and does not promote weight gain. Early small open-labeled studies showed improvement in biochemical markers $(p<0.0001)$ without any change in liver histology [Uygun et al. 2004; Marchensini et al. 2001]. A more recent randomized controlled trial comparing metformin ( $2 \mathrm{~g} /$ day) versus vitamin $\mathrm{E}$ (800 IU/day) or prescriptive diet showed an improvement in serum aminotransferase $(p=0.0013)$ levels if weight loss was present in any group, but a more significant decline was noted with metformin. This study also demonstrated a decrease in steatosis $(p=0.0004)$, necroinflammation $(p=0.012)$, and fibrosis $(p=0.012)$ in the metformin group [Bugianesi et al. 2005]. The exact potency of this medication is not entirely known and well-designed, large controlled trials with histopathologic endpoints are awaited. This agent may prove to be more useful in combination with other therapies, to include the thiazolidinediones.

\section{Lipid metabolism}

\section{3-hydroxy-3-methylglutaryl-coenzyme A reductase inhibitors}

This group of medications, more commonly known as statins (atorvastatin, pravastatin and simvastatin), have potent lipid lowering properties due to a disruption of a step in cholesterol biosynthesis. Initial concerns regarding safety in patients with NAFLD were disproven by multiple studies [Ekstedt et al. 2007; Lewis et al. 2007]. 
Studies using high-dose atorvastatin ( $80 \mathrm{mg} /$ day) showed an improvement in aminotransferase (11/24 normalized) levels and a possible decrease in steatosis [Gomez-Dominguez et al. 2006; Kiyici et al. 2003]. A more recent study with pravastatin $(80 \mathrm{mg} /$ day) showed a trend towards reduction in alanine aminotransferase [Lewis et al. 2007]. This class of medication has few studies to date and larger trials with high doses of the more potent atorvastatin may be useful.

\section{Ezetimibe}

This newer class of medication lowers cholesterol by blocking intestinal absorption. There have been two studies with mice looking at the effect on cholesterol and NAFLD. Both studies showed a decrease in alanine aminotransferase, and one study found a decrease in steatosis and fibrosis from baseline [Zheng et al. 2008; Deushi et al. 2007]. No clinical trials have been performed with this medication, but it may have benefit as either primary therapy or in combination with other agents.

Other

Probucol works by several mechanisms to include an increased fecal loss of bile acid-bound low-density lipoprotein cholesterol, decreased synthesis of cholesterol, and inhibition of enteral cholesterol absorption. It has also been shown to have strong antioxidant properties [Araki and Kitaoka, 2001] and tends to accumulate in fat. Merat et al. [2003] first evaluated this medication in a double-blind randomized controlled study of 30 patients. After 6 months of treatment, there was a normalization of alanine aminotransferase in $50 \%$ of the patients in the treatment arm. A follow-up study of 1 year of treatment showed a decrease in serum aminotransferase $(p=0.004$ and 0.001 ), and statistically significant improvement in steatosis and necroinflammation $(p=0.03)$, but not fibrosis $(p=0.79)$ [Merat et al. 2008]. Both studies were small in sample size, but show promise for treatment. This medication is currently not available in the United States.

Gemfibrozil has been evaluated in one small controlled trial that included 46 patients with NASH. After 4 weeks of therapy, there was a significant decline in alanine aminotransferase $(p<0.01)$. This effect was seen in patients with both elevated and normal triglyceride levels at baseline [Basaranoglu et al. 1999]. However, no studies involving histopathologic endpoints have been performed.

\section{Hepatoprotective medications}

\section{Antioxidants}

An increase in reactive oxygen species is thought to be a common pathway to hepatocellular injury and subsequent hepatic fibrosis. Vitamin $\mathrm{E}$ has been tried in many chronic disease states to reduce oxidative stress. In a controlled trial, vitamin E (800 IU daily) has been shown to improve insulin resistance, aspartate aminotransferase $(p=0.01)$ and alanine aminotransferase $(p=0.01)$ levels, and histological grade of steatosis $(p=0.01)$ over a 24 -week period. There was no change in necroinflammation or fibrosis, but a subset of patients with insulin resistance showed significant change in inflammation and peroxisome proliferator-activated receptor- $\alpha$ expression [Yakaryilmaz et al. 2007]. A double-blind, randomized, placebo-controlled trial of vitamin $\mathrm{E}$ (1000 IU/day) and vitamin C (1000 mg/day) in 49 patients over a 6-month period failed to show improvement in serum aminotransferase levels or histopathology when compared with placebo [Harrison et al. 2003]. Subsequently, Federico et al. [2006] studied vitamin E (360 mg/day) with silybin (376 mg/day), a natural flavonoid, and phosphatidylcholine $(776 \mathrm{mg} /$ day $)$ for 12 months in two groups of patients with NAFLD or chronic hepatitis $\mathrm{C}$ versus matched controls. Results showed improvement in alanine aminotransferase $(p<0.01)$, indices of liver fibrosis $(p<0.05)$, and steatosis by ultrasound imaging $(p<0.01)$. These positive effects were diminished at 12 months when compared with the 6-month evaluation. However, a metaanalysis of vitamin E use by Miller et al. [2005] showed that high dose ( $\geq 400 \mathrm{IU} /$ day) of vitamin E may increase all-cause mortality and should be avoided. Currently, it does not appear that vitamin $\mathrm{E}$ monotherapy has a role in NAFLD management. However, its efficacy and safety in combination therapy with other agents such as the thiazolidinedione class of medications is currently being studied in patients with NASH.

Betaine was first discovered in the juice of sugar beets and has been found to have protective properties by acting as an organic osmolyte and methyl donor. The latter is important to the proper function of the methionine cycle within the liver to maintain liver protein and hepatic 
fat metabolism [Craig, 2004]. A pilot study of 10 patients over a 12-month period showed a significant improvement in serum aspartate aminotransferase $(p=0.02)$ and alanine aminotransferase $(p=0.007)$ and marked improvement in steatosis $(p=0.10)$, necroinflammatory grade $(p=0.08)$, and stage of fibrosis $(p=0.04)$. Gastrointestinal side-effects were commonly reported but did not require dosage modification or discontinuation and were transient in nature [Abdelmalek et al. 2001]. Another larger, doubleblind, randomized, controlled trial of 191 patients receiving betaine with diethanolamine glucuronate and nicotinamide ascorbate versus placebo showed significant reductions in serum aminotransferases $\quad(p<0.001), \quad$ steatosis $(p<0.01)$, hepatomegaly $(p<0.05)$, and right upper quadrant pain $(p<0.001)$ [Miglio et al. 2000]. However, no follow-up liver biopsies were performed.

\section{Anti-inflammatory/antifibrotic}

Several groups of medication have been tried in an attempt to control the inflammatory and fibrogenic effects of the metabolic syndrome on NAFLD. Ursodeoxycholic acid was the first studied and showed beneficial results in a small double-blinded study by Santos et al. [2003]. A larger randomized trial by Lindor et al. [2004] used urosdeoxycholic acid (13-15 mg/kg/day) for a 2 -year period. This study showed no benefit over placebo for biochemical ( $p=0.85$ and 0.92) or histological markers; steatosis $(p=0.29)$, inflammation $(p=0.79)$, and fibrosis $(p=0.84)$. A more recent trial of 2 years compared three treatment groups; ursodeoxycholic acid and vita$\min \mathrm{E}$ (800 IU/day), urosdeoxycholic acid and placebo, or double placebo. There was improvement in alanine aminotransferase, aspartate aminotransferase, and steatosis in the dual therapy group, an improvement in alanine aminotransferase only in the ursodeoxycholic acid/placebo group, and no change in any factors in the control group [Dufour et al. 2006]. Based on these findings the use of ursodeoxycholic acid as monotherapy is not recommended at this time.

Pentoxifylline inhibits tumor necrosis factor- $\alpha$, with the potential to block some of the inflammatory insult on the liver. A pilot trial of 20 patients taking pentoxifylline $(1600 \mathrm{mg} /$ day $)$ for 12 months showed a significant decrease in serum aminotransferase levels ( $p=0.003$ and 0.002). However, nine patients did not complete the study due to nausea [Adams et al. 2004]. A more recent study using pentoxifylline $(1200 \mathrm{mg} /$ day $)$ in nine patients for 12 months showed it to be more tolerable with improvement in alanine aminotransferase $(p=0.003)$, steatosis ( $55 \%$ reduction), inflammation (55\% reduction), and fibrosis (4/6) [Satapathy et al. 2007]. Larger studies will be needed at various doses to determine the optimal dose and minimize the side effects.

Angiotensin receptor antagonists are currently being evaluated in large multi-centered trials based on previous small pilot trials and animal studies. It is well known that the renin-angiotensin system has an influence on key components of the metabolic syndrome, but also on hepatic stellate cells, the fibrogenic cells of the liver. Two animal model studies showed significant improvement in histological and biochemical parameters when an angiotensin receptor antagonist or angiotensin converting enzyme inhibitor was used [Toblli et al. 2008; Hirose et al. 2007]. A small uncontrolled study of seven patients taking losartan ( $50 \mathrm{mg} /$ day) for 48 weeks showed a benefit in serological markers for fibrosis, decreased hepatic necroinflammation (5/7) and fibrosis (4/7) on repeat liver biopsy [Yokohama et al. 2004]. These medications are routinely being used in patients with metabolic syndrome for various medical reasons and would have significant therapeutic benefits if current larger randomized trials show the same benefit in the treatment of NAFLD.

\section{Novel therapies}

There have been numerous studies evaluating the efficacy of alternative therapies over the last few years. In 2005 the use of diisopropylamine dichloroacetate, a herbal-derived chemical with both cellular proliferation and differentiation properties and vasodilatory effects [Kitamura et al. 1999], was used in a multicenter, randomized, double-blind controlled trial. The trial included 123 patients at two different doses of medication to evaluate safety as well as efficacy. Over 8 weeks, the normalization of alanine aminotransferase was noted in $55.7 \%$ of the low dose and $69.4 \%$ in the high dose; serum lipids improved in $67.2 \%$ and $67.7 \%$ respectively, and improvement in ultrasound steatosis grading was seen in $51.7 \%$ and $43.5 \%$ respectively. No statistical difference was noted between groups. There were no severe drug reactions reported and dry mouth was the only adverse reaction reported in $1.6 \%$ of the patients [Lu et al. 2005]. 
The Chinese herb YHK (YojoHenshiKo) has been shown to have antioxidant, hypoglycemic, antifibrotic and lipid-lowering properties. In the rat model, YHK has been shown to prevent NASH, promote weight loss, lower serum aminotransferases, and reduce visceral fat. It also promotes an increase in peroxisome proliferator-activated receptor- $\alpha$ and microsomal triglyceride transfer protein with a decrease in peroxisome proliferator-activated receptor- $\gamma$ mRNA contents [Stefano et al. 2008]. YHK has been demonstrated to be safe with a significant reduction in alanine aminotransferase $(p=0.036)$ in a small study of eight patients (five treatment) with NASH by Chande et al. [2006]. After discontinuation of the medication, there was an increase of alanine aminotransferase towards baseline after 4 weeks.

A 6-month open-label pilot study of folic acid did not show any improvement in serum aminotransferase levels $(p=0.5$ and 0.6$)$ in 10 patients with NASH [Charatcharoenwitthaya et al. 2007]. The study was based on folate deficiency causing a disturbance in hepatic methionine metabolism, which was shown to promote hepatic steatosis in the murine model [Rinella et al. 2008].

Resveratrol, a phytoalexin, is produced naturally by several plants, including the skin of red grapes, in response to attack by bacteria and fungi. It has shown to have antiviral, anticancer, neuroprotective, and anti-inflammatory properties in vitro. Recently, in vitro studies have shown improvement in triglyceride accumulation and insulin resistance by activating AMP-activated protein kinase. It is theorized that this activation can both treat and prevent the development of NALFD [Shang et al. 2008]. A recent observational study by Dunn et al. [2008] showed that modest wine consumption $(<10 \mathrm{~g} /$ day or 1 glass/day) was not only safe, but had a slight decrease in the prevalence of NAFLD with an odds ratio of 0.51 .

VSL\#3, a probiotic, may decrease inflammatory factors in patients with NAFLD and alcoholic liver cirrhosis [Loguercia et al. 2005]. A current study in phase 1 is looking at the efficacy and safety in patients with NAFLD. Most of these studies show promise for future treatment strategies. The progression to large, well-designed human studies would help define the true safety and efficacy of these therapies.

\section{Investigational therapies}

There are currently many investigational studies underway looking at alternative medications, longer duration of therapy, and combination therapy to enhance the response by effecting multiple pathways in the development of NAFLD and NASH. Many of these studies are currently being performed with pediatric patients, since the prevalence of NAFLD is increasing due to childhood obesity. A pilot study of acarbose for 12 weeks in the pediatric population is currently in phase II of investigation. Acarbose, an alphaglucosidase inhibitor like other medications for diabetes, has been shown to increase insulin sensitivity and increase adiponectin [Ochiai et al. 2008].

INT-747, an orally bioavailable FXR (farnesoid $\mathrm{X}$ receptor) agonist was first developed in 2001 and is currently being investigated for treatment of other forms of chronic liver disease and inflammatory bowel disease. FXR is a bile acid-responsive nuclear receptor that plays critical roles in the transcriptional regulation genes involved in cholesterol, bile acid, triglyceride and carbohydrate metabolism. The current trial is in phase II comparing two different doses with placebo. Another Chinese herb, berberine, is in phase II comparing 12 weeks of therapy against $15 \mathrm{mg}$ of pioglitazone and placebo [Kong et al. 2004].

A new line of medications known as the glitazars gave high hopes for the management of metabolic syndrome due to their beneficial effects on glucose and lipid metabolism. These medications were dual agonists for peroxisome proliferatoractivated receptor $\alpha$ and $\gamma$. Development was halted in 2005-2006 after a small increase in cardiovascular events. This has spurned the development of newer agents that are partial peroxisome proliferator-activated receptor $\gamma$ agonists. These medications work very similarly to the current thiazolidinediones, but with less adverse reaction, namely weight gain and edema. While these agents are currently in phase II trials, they may be a useful medication for NAFLD in the future.

\section{Conclusion}

Nonalcoholic fatty liver disease threatens to have a significant impact on our medical and economic systems. The understanding of the pathogenesis of NAFLD has aided in the implementation of medications used for the various treatments of the metabolic syndrome. 
This knowledge is also being used to further the development of more potent and safer medications that may be used to target NAFLD directly. Several medicinal as well as herbal therapies have shown promise and may be useful after further study in large, randomized, controlled trials as single-agent or multi-agent therapies.

Our recommended approach to treatment relies heavily on communication and education of our patients. The natural history of NAFLD, distinguishing between isolated fatty liver and NASH, and treatment options should all be discussed at initial consultation. If a liver biopsy is thought to be indicated [Harrison et al. 2008] to exclude $\mathrm{NASH}$ then one should be performed. For both isolated fatty liver and NASH, weight loss via diet and exercise remains the cornerstone of therapy. A goal of 7-10\% body weight loss should be targeted over 6-12 months. Weight-loss medication such as orlistat and/or surgical therapies such as Roux-en-Y and gastric banding may be discussed in the appropriate clinical situations. If no improvement in weight is noted over 3-6 months and the patient has NASH with a concerning amount of fibrosis, then pioglitazone may be considered. If initiated, then treatment for 12 months is recommended. During this time, reinforcement of good nutritional habits and exercise should continue with the hope that new habits have been formed by the time the pioglitazone is discontinued.

Ultimately in 2008, there remains no therapy that leads to complete regression of NASH in all cases (although bariatric surgery may come close), but current therapies do not leave us inept against this disease. We anxiously await newer therapies that target this disease without any significant associated side-effects. With larger, well-controlled trials, definitive therapies may be found. As the prevalence of NAFLD continues to increase, and primary preventive therapy falls short, it will be more important to find inexpensive, safe and effective medications before this chronic disease overburdens the resources of society.

\section{Conflict of interest statement}

None declared.

\section{References}

Abdelmalek, M., Angulo, P., Jorgensen, R., Sylvestre, P. and Lindor, K. (2001) Betaine, a promising new agent for patients with nonalcoholic steatohepatitis: results of a pilot study, $A m \mathcal{F}$ Gastroenterol 96: 2711-2717.

Adams, L., Zein, C., Angulo, P. and Lindor, K. (2004) A pilot trial of pentoxifylline in nonalcoholic steatohepatitis, Am $\mathcal{F}$ Gastroenterol 99: 2365-2368.

Aithal, G., Thomas, J., Kaye, P., Lawson, A., Ryder, S., Spendlove, I. et al. (2008) Randomized, placebo controlled trial of pioglitazone in non-diabetic subjects with nonalcoholic steatohepatitis (NASH), Gastroenterology 135: 1176-1184.

Anderson, T., Gluud, C., Franzmann, M. and Christofferen, P. (1991) Hepatic effects of dietary weight loss in morbidly obese subjects, $\mathcal{F}$ Hepatol 12 : 224-229.

Angelico, F., Burattin, M., Alessandri, C., Del Ben, M. and Lirussi, F. (2007) Drugs improving insulin resistance for non-alcoholic fatty liver disease and/or non-alcoholic steatohepatitis, Cochrane Database Syst. Rev. 1: CD005166.

Angulo, P., Hui, J., Marchesini, G., Bugianesi, E., George, J., Farrell, G. et al. (2007) The NAFLD fibrosis score: a noninvasive system that identifies liver fibrosis in patients with NAFLD, Hepatology 45: 846-854.

Araki, T. and Kitaoka, H. (2001) Antioxidative properties of probucol estimated by the reactivity with superoxide and by electrochemical oxidation, Chem Pharm Bull 49: 943-947.

Arslan, U., Turkoglu, S., Balcioglu, S., Tavil, Y., Karakan, T. and Cengel, A. (2007) Association between nonalcoholic fatty liver disease and coronary artery disease, Coron Artery Dis 18: 433-436.

Basaranoglu, M., Acbay, O. and Sonsuz, A. (1999) A controlled trial of gemfibrozil in the treatment of patients with nonalcoholic steatohepatitis (letter), f Hepatol 31: 384.

Belfort, R., Harrison, S., Brown, K., Darland, C., Finch, J., Hardies, J. et al. (2006) A placebo-controlled trial of pioglitazone in subjects with nonalcoholic steatohepatitis, N Engl f Med 355: 2297-2307.

Bergheim, I., Weber, S., Vos, M., Kramer, S., Volynets, V., Kaserouni, S. et al. (2008) Antibiotics protect against fructose-induced hepatic lipid accumulation in mice: role of endotoxin, $\mathcal{F}$ Hepatol 48: 983-992.

Browning, J., Szczepaniak, L., Dobbins, R., Nuremberg, P., Horton, J., Cohen, J. et al. (2004) Prevalence of hepatic steatosis in an urban population in the United States: impact of ethnicity, Hepatology 40: 1387-1395.

Bugianesi, E., Gentilcore, E., Manini, R., Natale, S., Vanni, E., David, E. et al. (2005) A randomized controlled trial of metformin versus vitamin $\mathrm{E}$ or prescriptive diet in nonalcoholic fatty liver disease, $A m \mathcal{F}$ Gastroenterol 100: 1082-1090.

Chande, N., Laidlaw, M., Adams, P. and Marotta, P. (2006) Yo Jyo Hen Shi Ko (YHK) improves 
transaminases in nonalcoholic steatohepatitis (NASH): a randomized pilot study, Dig Dis Sci 51: 1183-1189.

Charatcharoenwitthaya, P., Levy, C., Angulo, P., Keach, J., Jorgensen, R. and Lindor, K. (2007) Open-label pilot study of folic acid in patients with nonalcoholic steatohepatitis, Liver Int 27 : 220-226.

Craig, S.A. (2004) Betaine in human nutrition, $A m \mathcal{F}$ Clin Nutr 80: 539-549.

Das, K. and Kar, P. (2005) Update article: Non-alcoholic steatohepatitis, FAPI 53: 195-199.

Daubioul, C., Horsmans, Y., Lambert, P., Danse, E. and Delzenne, N. (2005) Effects of oligofructose on glucose and lipid metabolism in patients with nonalcoholic steatohepatitis: results of a pilot study, EFCN 59: 723-726.

Daubioul, C., Taper, H., De Wispelarera, L. and Delzenne, N. (2000) Dietary oligofructose lessens hepatic steatosis, but does not prevent hypertriglyceridemia in obese Zucker rats, $\mathcal{F}$ Nutr 130: 1314-1319.

Dehghan, M., Akhtar-Danesh, N. and Merchant, A. (2005) Childhood obesity, prevalence and prevention, Nutrit f 4: 24.

De Luis, D., Aller, R., Izaola, O., Sagrado, M., Conde, R., Gonzalez, J. et al. (2008) Effect of a hypocaloric diet in transaminases in nonalcoholic fatty liver disease and obese patients, relation with insulin resistance, Diabetes Res Clin Pract 79: 74-78.

Deushi, M., Nomura, M., Kawakami, A., Haraguchi, M., Ito, M., Okazaki, M. et al. (2007) Ezetimibe improves liver steatosis and insulin resistance in obese model of metabolic syndrome, FEBS Lett 581: 5664-5670.

Dixon, J., Bhathal, P. and O'Brien, P. (2006) Weight loss and non-alcoholic fatty liver disease: falls in gamma-glutamyl transferase concentrations are associated with histologic improvement, Obes Surg 16: 1278-1286.

Donnelly, K., Smith, C., Schwarzenberg, S., Jessurun, J., Boldt, M. and Parks, E. (2005) Sources of fatty acids stored in liver and secreted via lipoproteins in patients with nonalcoholic fatty liver disease, $\mathcal{F}$ Clin Invest 115: 1139-1142.

Dufour, J., Oneta, C., Gonvers, J., Bihl, F., Cerny, J., Cereda, J. et al. (2006) Randomized placebo-controlled trial of ursodeoxycholic acid with vitamine $\mathrm{E}$ in nonalcoholic steatohepatitis, Clin Gastroenterol Hepatol 4: 1537-1543.

Dunn, W., Xu, R. and Schwimmer, J. (2008) Modest wine drinking and decreased prevalence of suspected nonalcoholic fatty liver disease, Hepatology 47: 1947-1954.

Ebihara, K., Kusakabe, T., Hirata, M., Masuzaki, H., Miyanaga, F., Kobayahi, N. et al. (2007) Effiacy and safety of leptin-replacement therapy and possible mechanisms of leptin actions in patients with generalized lipodystrophy, $\mathcal{f}$ Clin Endocrinol Metab 92: $532-541$.

Ekstedt, M., Franzen, L., Mathiesen, U.,

Homqvist, M., Bodemar, G. and Kechagias, S. (2007) Statins in non-alcoholic fatty liver disease and chronically elevated liver enzymes: a histopathological followup study, f Hepatol 47: 135-141.

Federico, A., Trappoliere, M., Tuccillo, C., de Sio, I., Di Leva, A., Del Vecchio Blanco, C. et al. (2006) A new silybin-vitamin E-phospholipid complex improves insulin resistance and liver damage in patients with non-alcoholic fatty liver disease: preliminary observations, Gut 55: 901-902.

Gary-Bobo, M., Elachouri, G., Gallas, J., Janiak, P., Marini, P., Ravinet-Trillou, C. et al. (2007)

Rimonabant reduces obesity-associated hepatic steatosis and features of metabolic syndrome in obese zucker fa/fa rats, Hepatology 46: 122-129.

Gholam, P., Flanebaum, L., Machan, J., Charney, D. and Kutler, D. (2007) Nonalcoholic fatty liver disease in severely obese subjects, Am F Gastroenterol 102: 399-408.

Gomez-Dominguez, E., Gisbert, J., MorenoMonteagudo, J., Garcia-Buey, L. and MorenoOtero, R. (2006) A pilot study of atorvastatin treatment in dyslipemid, non-alcoholic fatty liver patients, Aliment Pharmacol Ther 23: 698-699.

Haque, M. and Sanyal, A. (2002) The metabolic abnormalities associated with nonalcoholic fatty liver disease, Best Pract Res Clin Gastroenterol 16: 709-731.

Harrison, S., Brunt, E. and Fecht, W. (2008) Orlistat in the treatment of overweight patients with nonalcoholic steatohepatitis (NASH): a multicentered, randomized prospective trial, Hepatology in press.

Harrison, S. and Day, C. (2007) Benefits of lifestyle modification in NAFLD, Gut 56: 1760-1769.

Harrison, S., Oliver, D., Arnold, H., Gogia, S. and Neuschwander-Tetri, B. (2008) Development and validation of a simple NAFLD clinical scoring system for identifying patients without advanced disease, Gut 57: 1441-1447.

Harrison, S., Torgerson, S. and Hayashi, P. (2003a) The natural history of nonalcoholic fatty liver disease: a clinical histopathological study, Am f Gastroenterol 98: 2042-2047.

Harrison, S., Torgerson, S., Hayashi, P., Ward, J. and Schenker, S. (2003b) Vitamin E and vitamin C improves fibrosis in patients with nonalcoholic steatohepatitis, Am f Gastroenterol 98: 2348-2350.

Harrison, S. (2008) Abnormal liver tests and fatty liver on ultrasound, Clin Gastro Hepatol 6: 26-29.

Hirose, A., Ono, M., Saibara, T., Nozaki, Y., Masuda, K., Yoshioka, A. et al. (2007) Angiotensin II type 1 receptor blocker inhibits fibrosis in rat nonalcoholic steatohepatitis, Hepatology 45: 1375-1381. 
Huang, M., Greenson, J., Chao, C., Anderson, L., Peterson, D., Jacobson, J. et al. (2005) One-year intesnse nutritional counseling results in histological improvement in patients with nonalcoholic steatohepatitis: a pilot study, Am F Gastroenterol 100: 1072-1081.

Huang, X., Fan, Y., Zhang, H., Wang, P., Yuan, J., Li, M. et al. (2008) Serum leptin and soluble leptin receptor in non-alcoholic fatty liver disease, World $\mathcal{F}$ Gastroenterol 14: 2888-2893.

Iijima, H., Moriyasu, F., Tsuchiya, K., Suzuki, S., Yoshida, M., Shimizu, M. et al. (2007) Decrease in accumulation of ultrasound contrast microbubbles in non-alcoholic steatohepatitis, Hepatol Res 37: 722-730.

Joy, D., Thava, V. and Scott, B. (2003) Diagnosis of fatty liver disease: is biopsy necessary? Eur $\mathcal{F}$ Gastroenterol Hepatol 15: 539-543.

Kitamura, N., Ota, Y. and Mimura, K. (1999) Effects of diisopropylamine dichloroacetate on proliferation and differentiation of normal human keratinocytes in vitro, Skin Parmachology and Applied Skin Physiology 12: 317-325.

Kiyici, M., Gulten, M., Gurel, S., Nak, S., Dolar, E., Savci, G. et al. (2003) Ursodeoxycholic acid and atorvastatin in the treatment of nonalcoholic steatohepatitis, Can $\mathcal{F}$ Gastroenterol 17: 713-718.

Kong, W., Wei, J., Abidi, P., Lin, M., Inaba, S., Li, C. et al. (2004) Berberine is a novel cholesterol-lowering drug working through a unique mechanism distinct from statins, Nat Med 10: 1344-1351.

Le, K., Tappy, L. and D’Alessio, D. (2007)

Mitochondrial dysfunction and insulin resistance: a matter of lifestyle?, Curr Opin Nutr Metab Care 10: 494-497.

Leevy, C. (1962) Fatty liver: A study of 270 patients with biopsy proven fatty liver and a review of the literature, Medicine 41: 249-278.

Lewis, J., Mortensen, M., Zweig, S., Fusco, M., Medoff, J. and Belder, R. (2007) Efficacy and safety of high-dose pravastatin in hypercholesterolemic patients with well-compensated chronic liver disease: results of a prospective, randomized, double-blind, placebocontrolled, multicenter trial, Hepatology 46: 1453-1463.

Lindor, K., Kowdley, K., Heathcote, E., Harrison, M., Jorgensen, R., Angulo, P. et al. (2004)

Ursodeoxycholic acid for treatment of nonalcoholic steatohepatitis: results of a randomized trial, Hepatology 39: 770-778.

Loguercia, C., Federico, A., Tuccillo, C., Terracciano, F., D’Auria, M., De Simone, C. et al. (2005) Beneficial effects of a probiotic VSL\#3 on parameters of liver dysfunction in chronic liver disease, f Clin Gastroenterol 39: 540-543.

Lu, L., Zeng, M., Mao, Y., Chen, C., Fu, Q., Wang, J., Xie, W. et al. (2005) Diisopropylamine dichloroacetate in the treatment of nonalcoholic fatty liver disease: a multicenter random double-blind controlled trial, Chinese f Hepatol 13: 92-95.

Lutchman, G., Promrat, K., Kleiner, D., Heller, T., Ghany, M., Yanovski, J. et al. (2006) Changes in serum adipokine levels during pioglitazone treatment for nonalcoholic steatohepatitis, relationship to histologic improvement, Clin Gastroenterol Hepatol 4: 1048-1052.

Machado, M., Marques-Vidal, P. and Cortez-Pinto, H (2006) Hepatic histology in obese patients undergoing bariatric surgery, $\mathcal{f}$ Hepatol 45: 600-606.

Manning, D. and Afdhal, N. (2008) Diagnosis and quantitation of fibrosis, Gastroenterology 134: 1670-1681.

Marchensini, G., Bianchi, G., Tomassetti, S., Zoli, M. and Melchionda, N. (2001) Metformin in non-alcoholic steatohepatitis, Lancet 348: 893-894.

Marubbio, A., Buchwald, H., Schwartz, H. and Varco, R. (1976) Hepatic lesions of central pericellular fibrosis in morbid obesity and after jejunoileal bypass, Am 7 Clin Pathol 66: 684-691.

Mattar, S., Velcu, L., Rabinovitz, M., Krasinskas, A., Barinas-Mitchell, M., Eid, G. et al. (2005) Surgicallyinduced weight loss significantly improved nonalcoholic fatty liver disease and the metabolic syndrome, Ann Surg 242: 610-620.

Mendez-Sanchez, N., Gonzalez, V., Chavez-Tapia, N., Ramos, M. and Uribe, M. (2004) Weight reduction and ursodeoxycholic acid subjects with nonalcoholic fatty liver disease. A double-blind, placebo controlled trial, Ann Hepatol 3: 108-112.

Merat, S., Aduli, M., Kazemi, R., Sotoudeh, M., Sedighi, N., Sohrabi, M. et al. (2008) Liver histology changes in nonalcoholic steatohepatitis after one year of treatment with probucol, Dig Dis Sci 53: 2246-2250.

Merat, S., Malekzadeh, R., Sohrabi, M., Sotoudeh, M., Rakhshani, N., Sohrabpour, A. et al. (2003) Probucol in the treatment of non-alcoholic steatohepatitis: a double-blind randomized controlled study, f Hepatol 38: 414-418.

Miglio, F., Rovati, L., Santoro, A. and Setnikar, I. (2000) Efficacy and safety of oral betaine glucuronate in non-alcoholic steatohepatitis. A double-blind, randomized, parallel-group, placebo-controlled prospective clinical study, Arzneimittelforschung 50: 722-727.

Miller, E., Pastor-Barriuso, R., Dalal, D., Riemersma, R., Appel, L. and Guallar, E. (2005) Meta-analysis: high-dosage vitamin E supplementation may increase all-cause mortality, Ann Intern Med 142: 37-46.

Mottin, C., Moretto, M., Padoin, A., Swarowsky, A., Toneto, M., Glock, L. et al. (2004) The role of ultrasound in the diagnosis of hepatic steatosis in morbidly obese patients, Obes Surg 14: 635-637. 
Neuschwander-Tetri, B.A. and Bacon, B. (1996) Nonalcoholic steatohepatitis, Med Clin North Am 80: 1147-1166.

Ochiai, H., Ooka, H., Shida, C., Ishikawa, T., Inoue, D. and Okazaki, R. (2008) Acarbose treatment increases serum total adiponectin levels in patients with type 2 diabetes, Endocr F 55: 549-556.

Oral, E., Simha, V., Ruiz, E., Anderwelt, A., Premkumar, A., Snell, P. et al. (2002) Leptin-replacement therapy for lipodystrophy, $N$ Engl F Med 346: 570-578.

Ouyang, X., Cirillo, P., Sautin, Y., McCall, S., Bruchette, J., Diehl, A. et al. (2008) Fructose consumption as a risk factor for nonalcoholic fatty liver disease, f Hepatol 48: 993-999.

Parsons, T., Power, C. and Summerbell, C. (1999) Childhood predictors of adult obesity: a systematic review, Int $\mathcal{F}$ Obesity 23: S1-S107.

Ratziu, V., Giral, P., Jacqueminet, S., Charlotte, F., Hartemann-Heurtier, A., Serfaty, L., et al. (2008) Rosiglitazone for nonalcoholic steatohepatitis: Oneyear results of the randomized placebo-controlled fatty liver improvement with rosiglitazone therapy (FLIRT) trial, Gastroenterology 135: 100-110.

Ratziu, V., Massard, J., Charlotte, F., ImbertBismut, F., Bonyhay, L., Tahiri, M. et al. (2006) Diagnostic value of biochemical markers (FibroTestFibroSURE) for the prediction of liver fibrosis in patients with non-alcoholic fatty liver disease, $B M C$ Gastroenterol 14: 6-34.

Rinella, M., Elias, M., Smolak, R., Fu, T., Borensztajn, J. and Green, R. (2008) Mechanisms of hepatic steatosis in mice fed a lipogenic methionine choline-deficient diet, $\mathcal{F}$ Lipid Res 49: 1068-1076.

Ryan, M., Abbasi, F., Lamendola, C., Carter, S. and McLaughlin, T. (2007) Serum alanine aminotransferase levels decrease further with carbohydrate than fat restriction in insulin-resistant adults, Diabetes Care 30: 1075-1080.

Sabuncu, T., Nazligul, Y., Karaoglanoglu, M., Ucar, E. and Kilic, F. (2003) The effects of sibutramine and orlistat on the ultrasonographic findings, insulin resistance and liver enzyme levels in obese patients with non-alcoholic steatohepatitis, Rom $\mathcal{F}$ Gastroenterol 12: 189-192.

Santos, V., Lanzoni, V., Szenjnfeld, J., Shigueoka, D. and Parise, R. (2003) A randomized double-blind study of the short-time treatment of obese patients with nonalcoholic fatty liver disease with ursodeoxycholic acid, Brazilian f Med Bio Res 36: 723-729.

Sanyal, A., Mofrad, P., Contos, M., Sargeant, C., Luketic, V., Sterling, R. et al. (2004) A pilot study of vitamin $\mathrm{E}$ versus vitamin $\mathrm{E}$ and pioglitazone for the treatment of nonalcoholic steatohepatitis, Clin Gastroenterol Hepatol 2: 1107-1115.

Satapathy, S., Sakhuja, P., Malhotra, V., Sharma, B. and Sarin, S. (2007) Beneficial effects of pentoxifylline on hepatic steatosis, fibrosis and necroinlammation in patients with non-alcoholic steatohepatitis, $\mathcal{F}$ Gastroenterol Hepatol 22: 634-638.

Screenivasa Baba, C., Alexander, G., Kalyani, B., Pandey, R., Rastogi, S., Pandey, A. et al. (2006) Effect of exercise and dietary modification on serum aminotransferase levels in patients with nonalcoholic steatohepatitis, $\mathcal{F}$ Gastroenterol Hepatol 21: 191-198.

Shang, J., Chen, L., Xiao, F., Sun, H., Ding, H. and Xiao, H. (2008) Resveratrol improves non-alcoholic fatty liver disease by activating AMP-activated protein kinase, Acta Pharmacol Sin 29: 698-706.

Stefano, J., de Oliveira, C., Correa-Giannella, M., de Lima, V., de S'a, S., de Oliveira, E. et al. (2008) Nonalcholic steatohepatitis (NASH) in OB-OB mice treated with YHK (YojoHenshiKo): effects on peroxisome proliferator-activated receptors (PPARs) and microsomal triglyceride transfer protein (MTP), Med f 27: 227-234.

Suzuki, A., Lindor, K., St Saver, J., Lymp, J., Mendez, F., Muto, A. et al. (2005) Effect of changes on body weight and lifestyle in nonalcoholic fatty liver disease, $\mathcal{F}$ Hepatol 43: 1060-1066.

Targher, G., Bertonlini, L., Poli, F., Rodella, S., Scala, L., Tessari, R. et al. (2005) Nonalcoholic fatty liver disease and risk of future cardiovascular events among type 2 diabetic patients, Diabetes 54: 3541-3546.

Thomas, E., Brynes, A., Hamilton, G., Patel, N., Spong, A., Goldin, R. et al. (2006) Effect of nutritional counseling on hepatic, muscle, and adipose fat tissue contentand distribution in nonalcoholic fatty liver disease, World f Gastroenterol 12: 5813-5819.

Toblli, J., Munoz, M., Cao, G., Mella, J., Pereyra, J. and Mastai, R. (2008) ACE inhibition and AT1 receptor blockade prevent fatty liver and fibrosis in obese Zucker rats, Obesity 16: 770-776.

Tushuizen, M., Bunck, M., Pouwels, P., van Waesberghe, J., Diamant, M. and Heine, R. (2006) Incretin mimetics as a novel therapeutic option for hepatic steatosis, Liver Int 26: 1015-1017.

Uygun, A., Kadayifci, A., Isik, A., Ozgurtas, T., Deveci, S., Tuzun, A. et al. (2004) Metformin in the treatment of patients with non-alcoholic steatohepatitis, Aliment Pharmacol Ther 19: 537-544.

van der Poorten, D., Milner, K., Hui, J., Hodge, A., Trenell, M., Kench, J. et al. (2008) Visceral fat: A key mediator of steatohepatitis in metabolic liver disease, Hepatology 48: 449-457.

Wang, R. and Koretz, R. (2003) Is weight reduction an effective therapy for nonalcoholic fatty liver disease? A systematic review, $\mathrm{Am} \mathcal{F} \mathrm{Med}$ 115: 554-559.

Wanless, I. and Lentz, J. (1990) Fatty liver hepatitis (steatohepatitis) and obesity: an autopsy study with analysis of risk factors, Hepatology 12: 1106-1110.

Weisberg, S., McCann, D., Desai, M., Rosenbaum, M., Leibel, R. and Ferrant, A. (2003) 
Obesity is associated with macrophage accumulation in adipose tissue, $\mathcal{F}$ Clin Invest 112: 1796-1808.

Whitaker, R., Wright, J., Pepe, M., Seidel, K. and Dietz, W. (1997) Predicting obesity in young adulthood from childhood and parental obesity, $N$ Engl $\mathcal{F}$ Med 337: 869-873.

Wong, V., Wong, G., Chim, A., Tse, A., Tsang, S., Hui, A. et al. (2008) Validation of NAFLD fibrosis score in a Chinese population with low prevalence of advanced fibrosis, Am F Gastroenterol 103: $1682-1688$.

World Health Organization (2006) Fact Sheet No. 311. WHO, Geneva.

Xu, A., Wang, Y., Keshaw, H., Xu, L.Y., Lam, K.S., Cooper, G.T. (2003) The fat-derived hormone adiponectin alleviates alcoholic and nonalcoholic fatty liver diseases in mice, $\mathcal{F}$ Clin Invest 112: 91100.

Yakaryilmaz, F., Guliter, S., Savas, B., Erdem, O., Ersoy, R., Erden, E. et al. (2007) Effects of vitamin E treatment on peroxisome proliferator-activated receptor-[alpha] expression and insulin resistance in patients with non-alcoholic steatohepatitis: results of a pilot study, Intern Med F 37: 229-235.
Yokohama, S., Yoneda, M., Haneda, M., Okamoto, S., Okada, M., Aso, K. et al. (2004) Therapeutic efficacy of an angiotensin II receptor antagonist in patients with nonalcoholic steatohepatitis, Hepatology 40: 1222-1225.

Zelber-Sagi, S., Kessler, A., Brazowsky, A., Webb, M., Lurie, Y., Santo, M. et al. (2006) A double-blind randomized placebo controlled trial of orlistat for treatment of nonalcoholic fatty liver disease, Clin Gastroenterol Hepatol 4: 639-644.

Zelber-Sagi, S., Nitzan-Kaluski, D., Goldsmith, R., Webb, M., Blendis, L., Helpern, Z. et al. (2008) Long term nutritional intake and the risk for nonalcoholic fatty liver disease (NAFLD): a population based study, f Hepatol 47: 711-717.

Zheng, S., Hoos, L., Cook, J., Tetzloff, G., Davis, H., van Heek, M. et al. (2008) Ezetimibe improves high fat and cholesterol diet-induced non-alcoholic fatty liver disease in mice, Eur $\mathcal{F}$ Pharmacol 584: 118-124.

Zivkovic, A., German, J. and Sanyal, A. (2007) Comparative review of diets for the metabolic syndrome: implications for nonalcoholic fatty liver disease, Am f Clin Nutr 86: 282-300.
Visit SAGE journals online http://tag.sagepub.com

(SSAGEJOURNALS 